\title{
Structures and magnetic properties of iron chains encapsulated in tubal carbon nanocapsules
}

\author{
N. Fujima \\ Faculty of Engineering, Shizuoka University, Hamamatsu 432-8561, Japan \\ T. Oda \\ Department of Computational Science, Faculty of Science, Kanazawa University, Kanazawa 920-1192, Japan \\ (Received 25 February 2004; revised manuscript received 15 December 2004; published 14 March 2005)
}

\begin{abstract}
Structures and magnetic properties of $\mathrm{Fe}_{n}$ chains encapsulated in tubal carbon nanocages, $\mathrm{C}_{10(n+1)}(n$ $=1-4)$ and $\mathrm{C}_{48}(n=1,2)$, are studied by means of the first-principles approach of noncollinear magnetism, in which the atomic and magnetic structures can be optimized simultaneously and self-consistently. The optimizations show that the globular capsule $\mathrm{FeC}_{20}$ is energetically unfavorable, while the longer capsule $\mathrm{Fe}_{n} \mathrm{C}_{10(n+1)}$ for $n=2-4$ becomes favorable and retains the linear structure of the iron chain along the center axis of the cages. The spin magnetic moments of the Fe atoms align in antiparallel for $n=2$ and 4 in contrast with the parallel magnetic moments in bare $\mathrm{Fe}_{n}$ chains. These antiferromagnetic configurations are stabilized by effective antiferromagnetic interactions induced by the carbon cages, where the small magnetic moments appear in the same orientation as those of the adjacent $\mathrm{Fe}$ atoms. For thicker capsules $\mathrm{Fe}_{n} \mathrm{C}_{48}, \mathrm{Fe}$ atoms also settle along the center axis of the cage. Due to the decrease of interaction between $\mathrm{Fe}$ and $\mathrm{C}$ atoms, the parallel alignment of magnetic moments on $\mathrm{Fe}$ atoms is more stabilized than the antiparallel one. For both the cages $\mathrm{C}_{10(n+1)}$ and $\mathrm{C}_{48}$, terminal effects play a certain role in the settlement of $\mathrm{Fe}$ atoms along the center axis and the antiferromagnetic arrangement.
\end{abstract}

DOI: 10.1103/PhysRevB.71.115412

PACS number(s): 73.22.-f, 75.75.+a, 61.46.+w, 36.40.Cg

\section{INTRODUCTION}

The numerous potential applications of carbon nanotubes and nanocapsules have attracted the interest of many researchers and engineers. ${ }^{1}$ Carbon materials, not only in themselves but also those used as wrapping materials, have become the focus of research attention ${ }^{2,3}$ because of their physical flexibility and chemical stability. It is known that transition-metal (TM) nanoparticles can be well contained in carbon nanocapsules or nanotubes, and that they (particularly $\mathrm{Ni}, \mathrm{Co}, \mathrm{Fe}$, and their alloys) can catalyze synthesis of carbon nanotubes. ${ }^{4,5}$ Then the physical and chemical properties of TMs in carbon materials are certainly of great interest for developing new hybrid materials or understanding synthetic mechanisms. The simplest materials to understand their properties may be single-wall nanotubes (SWNTs) or capsules (SWNCs) with a TM particle. There have been some experimental studies about SWNTs or multiwall nanotubes filling with TM atoms. ${ }^{6-11}$ However, only a few theoretical works $^{12-15}$ related to SWNTs or SWNCs with TM atoms have been reported although extensive studies have been performed on the interactions between TM atoms and graphite or graphene ${ }^{16-19}$ where no curvature or pentagonal ring exists, unlike in nanotube or nanocapsules. A complete understanding of the magnetism of TM particles in SWNTs and SWNCs or interaction between them still remains a challenging problem.

TM nanoparticles themselves are interesting as lowdimensional magnetic materials where large magnetic moments, anomalous magnetic arrangements, etc., are expected to appear. $^{20-23}$ The authors ${ }^{24-27}$ and other researchers ${ }^{28,29}$ have theoretically studied magnetism of TM clusters such as $\mathrm{Fe}, \mathrm{Mn}$, and $\mathrm{Cr}$ by the noncollinear spin density functional method, and found that some noncollinear magnetic mo- ments appears in certain sizes and geometries of the clusters. The authors also found that Fe linear chains $(n<7)$ change the magnetic arrangement from collinear to noncollinear and vice versa with change of the interatomic distances. ${ }^{27}$

This magnetism is very interesting; however, such small clusters, especially linear chain clusters, easily change their atomic and magnetic structures depending on their environments. In order to apply the functions or the properties of these clusters, it is necessary to find containers or supporting materials that keep or emphasize their structures and properties or prevent oxidation. The SWNT or SWNC is one of the best candidates to contain or adsorb TM materials. Therefore, it is important to research their magnetic properties and atomic structures in or on them.

In the present paper, we calculate the electronic and atomic structures of the $\mathrm{Fe}_{n}$ chains in carbon tubal cages $\mathrm{C}_{10(n+1)}$ for $n<5$ by first-principles molecular dynamics. There may exist a wide variety of possible magnetic arrangements on the capsules. Therefore we perform a noncollinear calculation in order that magnetic moments are selfconsistently determined without restriction of the magnitude and the direction. We indicate that the Fe linear chains are stabilized in the capsule, and that the magnetic moments align in antiparallel for $n=2$ and 4 while all the bare chains have parallel magnetic alignment. It is also indicated that some induced magnetic moments appear on the carbon cages, especially on the terminal pentagons.

To see the effect of the size of cage, we also calculate the electronic and atomic structures of $\mathrm{Fe}_{n} \mathrm{C}_{48}$ for $n=1$ and 2, and compare the positions and magnetic properties of $\mathrm{Fe}$ atoms in the large cage $\mathrm{C}_{48}$ with those in the small cage $\mathrm{C}_{10(n+1)}$. These cages have a diameter of $4-7 \AA$, which is smaller than that of abundantly observed SWNTs, ${ }^{1}$ while the diameter of $3 \AA$ has been reported in multiwall tubes. ${ }^{30}$ 


\section{CALCULATIONS}

\section{A. Methods}

All the calculations in the present work are performed by first-principles molecular dynamics, ${ }^{24,31}$ in which a generalized spin-density functional method is employed so that noncollinear spin densities are obtained. ${ }^{32}$ The electronic wave functions and the atomic positions are simultaneously optimized by solving the equations of motion without any restriction for atomic positions or magnetic arrangement. The core orbitals, $1 s$ for $\mathrm{C}$ and $1 s-2 p$ for $\mathrm{Fe}$, are expressed with the ultrasoft pseudopotential scheme, ${ }^{33,34}$ where hard components augment the electron density. The wave functions and the charge- and spin-density matrices are expanded into a set of plane waves with cutoff energies of 24 and 250 Ry, respectively. The generalized gradient exchange-correlation potential $^{35}$ is applied to the locally oriented spin densities and their gradients. ${ }^{36}$ The capsules are set on a diagonal of a simple cubic unit cell with a lattice constant of 20 a.u. Details of the scheme are described in Refs. 37-39.

The calculations have been performed in a noncollinear scheme; however, the results for all the capsules indicate that the magnetic moments align only collinearly. Then the density of states is calculated in the usual spin-polarized scheme. It is noted that the noncollinear calculation has a certain advantage over the collinear calculation even if the final state becomes collinear. The collinear calculation is often entangled in artificial metastable states in complicated magnetic systems while the noncollinear calculation can easily pass through such states without restriction on the orientation of the spin density.

Atomic spin magnetic moments are estimated by integrating spin densities within a radius of $0.90 \AA$ for a Fe atom and $0.69 \AA$ for a $\mathrm{C}$ atom. For example, the atomic magnetic moment of an $\mathrm{Fe}$ atom [see Fig. 2(a) below] shows a value of $3.24 \mu_{\mathrm{B}}$ while the whole magnetic moment in the cell reaches $4.0 \mu_{\mathrm{B}}$, so about $80 \%$ of the $3 d$ magnetic component is included within the radius.

\section{B. Atomic structures}

All the atomic positions of the capsules are fully optimized from the following initial atomic structure. For the $\mathrm{Fe}_{n} \mathrm{C}_{10(n+1)}$ capsules, a SWNT that is terminated with two carbon pentagons is employed as the cage. The employed SWNT is the zigzag type with a chiral vector of $(5,0)$ (Ref. 1) and a radius of $1.96 \AA$. Fe atoms are located along the center axis of the SWNC. Ten carbon atoms form a zigzag circle surrounding each Fe atom, that is, an Fe atom is in the middle of ten $\mathrm{C}-\mathrm{C}$ bonds. Fagan et al. have reported in their calculations for an $\mathrm{Fe}$ atom in an $(8,0)$ SWNT that the middle of the $\mathrm{C}-\mathrm{C}$ bond is energetically more favorable than the center of a hexagon for an $\mathrm{Fe}$ atom, and that the interatomic distance between $\mathrm{Fe}$ and $\mathrm{C}$ is $2 \AA^{14}$ In the initial arrangement, the interatomic distance between the adjacent $\mathrm{C}$ and $\mathrm{Fe}$ atoms is $1.99 \AA$, and the intervals of the $\mathrm{Fe}$ atoms are $2.13 \AA$, corresponding to the period of the SWNT. It is noted that the interval of $\mathrm{Fe}$ atoms is close to the interatomic distance of bare small Fe clusters $(2.0-2.3 \AA) .{ }^{20,21,24,28}$
There are five pentagonal rings adjacent to the terminal pentagon so that three pentagons meet at a vertex. Then it is expected that $s p^{3}$-like orbitals appear and that some of them make dangling bonds. Meanwhile, there exist slightly curved hexagonal rings on the middle of the cage, and $s p^{2}$-like orbitals with $\pi$ bonds are expected to be dominant.

For the thicker capsules, $\mathrm{Fe}_{n} \mathrm{C}_{48}(n=1,2)$, the carbon cage consists of a zigzag type $(8,0)$ SWNT terminated by octagonal rings, which are surrounded by eight pentagons. Because the terminal ring is not a pentagon but an octagon, the $s p^{3}$-like character of the orbitals at the ends is less than for the $\mathrm{Fe}_{n} \mathrm{C}_{10(n+1)}$. The radius of the capsule is $3.14 \AA$. In this capsule, several initial positions of $\mathrm{Fe}$ atom and dimer are examined.

\section{RESULTS}

Before discussing the atomic and magnetic structures of individual capsules, we indicate comprehensive results of all the capsules of $\mathrm{Fe}_{n} \mathrm{C}_{10(n+1)}$. Figures 1(a)-1(d) show the atomic structures and spin magnetic moments of $\mathrm{Fe}_{n} \mathrm{C}_{10(n+1)}$ for $n=1-4$. In the figures, the interatomic distances between $\mathrm{Fe}$ atoms are shown above the atomic bonds in angstroms and the absolute magnetic moments of Fe atoms below the figures in $\mu_{\mathrm{B}}$. The net magnetic moments of the whole capsules are also shown in parentheses. The dimensions of the carbon cages are shown in the upper left-hand side; the absolute magnetic moments of $\mathrm{C}$ atoms are in the right-hand side. The arrows indicate the direction of the magnetic moment.

These results, as well as some energy estimations, the total energy differences $E_{\text {diff }}$ and the binding energy between the Fe chains and cages, $E_{\mathrm{b}}$, are summarized in Table I. $E_{\mathrm{b}}$ are calculated as $E_{\mathrm{b}}=\left(E_{\mathrm{Fe}}+E_{\mathrm{C}}\right)-E_{\mathrm{FeC}}$, where $E_{\mathrm{Fe}}, E_{\mathrm{C}}$, and $E_{\mathrm{FeC}}$ are the total energies of the bare $\mathrm{Fe}_{n}$ chain, the empty cage $\mathrm{C}_{10(n+1)}$, and the hybrid capsule $\mathrm{Fe}_{n} \mathrm{C}_{10(n+1)}$, respectively. In this energy calculation, the atomic structures of the chains and cages are fixed to the optimized structure of the hybrid capsule.

We have examined the accuracy of these energies by changing the cutoff energy from 24 to 30 Ry and the length of the unit cell from 20 to 25 a.u. This examination shows that the accuracy of energies is about $\pm 0.05 \mathrm{eV}$.

Other magnetic alignments with slightly higher total energies are listed as $\left(\mathrm{b}^{\prime}\right)$ and $\left(\mathrm{d}^{\prime}\right)$ for $n=2$ and 4 . (For these two alignments, the atomic positions are optimized under the collinear restriction.)

The atomic magnetic moments of the fixed bare chains (the free-standing linear chains whose atomic geometries are fixed at the optimized ones in the cages), $m_{\text {bare }}$, are also listed in the table. The difference between the magnetic moments $m$ and $m_{\text {bare }}$ indicates the explicit effect of the carbon cages.

Figures 2(a)-2(d) provide the atomic structure and magnetic moments of entirely free Fe chains in a vacuum, calculated by the same method. The comparison of these data with those in Fig. 1 may reveal geometrical confinement effects of cages.

The total density of states (DOS) and partial density of states (PDOS) for the $3 d$ component of the $\mathrm{Fe}$ atoms are 


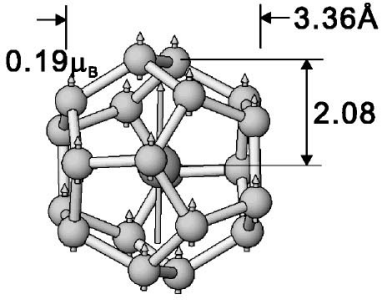

(a) $3.31\left(8.00 \mu_{\mathrm{B}}\right)$

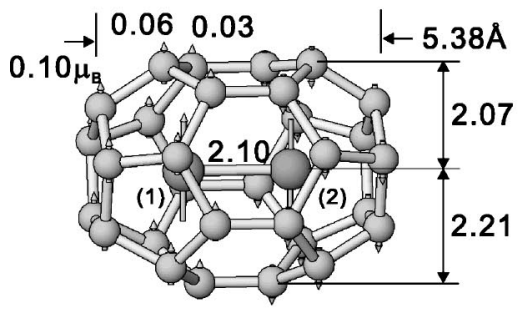

(b)

$2.38-2.38\left(0.00 \mu_{B}\right)$
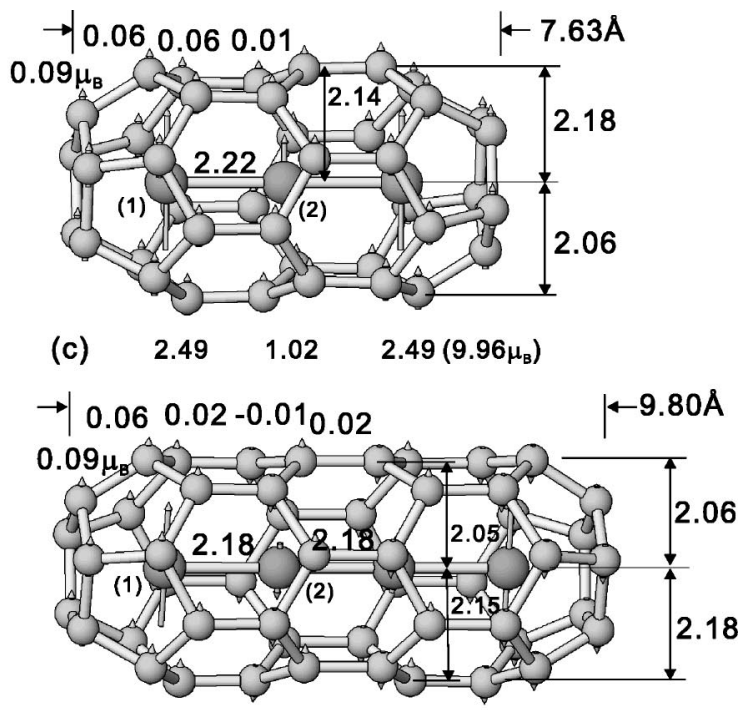

(d)
$2.55 \quad-0.29$
0.29
FIG. 1. Structures and magnetic moments of $\mathrm{Fe}_{n} \mathrm{C}_{10(n+1)}, n=$ (a) 1, (b) 2, (c) 3, and (d) 4. The magnetic moments $\left(\mu_{\mathrm{B}}\right)$ of $\mathrm{Fe}$ atoms are listed below the figures. The net magnetic moment of the whole capsule is shown in parentheses. The magnetic moments of the $\mathrm{C}$ cages are indicated on the upper left side, and the dimensions on the right side. The interatomic distances between $\mathrm{Fe}$ atoms $(\AA)$ are indicated above the atomic bonds. shown in Figs. 3(a)-3(d), corresponding to Figs. 1(a)-1(d). The gray and black curves indicate the DOS and PDOS, respectively. The PDOSs for different atomic sites are distinguished by solid and dashed curves, and multiplied by some factors for clarity. The upper (lower) side shows the spin-up (-down) components. The vertical dash-dotted line indicates the highest occupied level $\left(E_{\mathrm{F}}\right)$. The DOS curves are obtained by replacing the discrete level $E_{i}$ with the Gaussian function $e^{-\left(E-E_{i}\right)^{2} / 2 \sigma^{2}}$ with $\sigma$ of $0.1 \mathrm{eV}$. The PDOSs are estimated by projecting the wave function onto the $1 m$ components within a radius of $1.26 \AA$ around the $\mathrm{Fe}$ atom, and summing up the $m$ components of $l=2$. It is noted that this radius fully covers core and semicore orbitals but only partially the valence orbitals.

\section{A. $\mathrm{FeC}_{20}$}

Figure 1(a) shows the optimized atomic structure with the magnetic moments of $\mathrm{FeC}_{20}$. The optimized structure keeps the initial structure, the regular dodecahedron, as shown in the figure. The interatomic distance between the central $\mathrm{Fe}$ and surrounding $\mathrm{C}$ atoms is $2.11 \AA$, increasing by $7 \%$ from the initial distance $(1.99 \AA)$.
The magnetic moment of the Fe atom is $3.31 \mu_{\mathrm{B}}$. Although this value is underestimated because of the limitation of the radius in the integral of the spin density, this is still similar to the typical value in the electronic configuration of a $\mathrm{Fe}$ atom, $3 d_{\uparrow}^{5} 3 d_{\downarrow}^{1}$.

The magnetic moment in the $\mathrm{Fe}$ atom induces a small magnetic moment of $0.19 \mu_{\mathrm{B}}$ /atom in surrounding $\mathrm{C}$ atoms. All the magnetic moments align in parallel. The total magnetic moment of the capsule is $8.0 \mu_{\mathrm{B}}$, twice the moment of the bare Fe atom. It is noted that the induced magnetic moments on the terminal pentagons gradually decrease with increasing capsule length as shown in Figs. 1(a)-1(d).

The binding energy in Table I is negative for $n=1$, that is, the capsule $\mathrm{FeC}_{20}$ is energetically unfavorable. (It changes to positive with increasing capsule length.)

The DOS and PDOS curves in Fig. 3(a) well explain the magnetic moment and energetic unfavorableness of $\mathrm{FeC}_{20}$. The optimization shows that the symmetry of $\mathrm{FeC}_{20}$ is almost $I_{h}$; hence, the $3 d$ orbitals are almost degenerate as $h_{g}$-like states. Then, the two prominent peaks of the PDOS [solid curve in Fig. 3(a)] are assigned to the bonding and antibonding states with the $2 s$ or $2 p$ orbitals of the surrounding $\mathrm{C}$ atoms. On the spin-up side, both the peaks lie under $E_{\mathrm{F}}$. So the $3 d$-electron population is almost 5 and the occu-

TABLE I. Total-energy differences $E_{\text {diff }}$, binding energies $E_{\mathrm{b}}$, interatomic distances of $\mathrm{Fe}$ atoms, $d$, and magnetic moments of $\mathrm{Fe}$ atoms, $m$, for $\mathrm{Fe}_{n} \mathrm{C}_{10(n+1)}$ capsules. The rows (a)-(d) correspond to Figs. 1 and 3(a)-3(d). Rows 2(b') and 4(d') show slightly higher-energy states. Magnetic moments of bare $\mathrm{Fe}_{n}$ with the same interatomic distances as those in the cages, $m_{\text {bare }}$, are also listed as references.

\begin{tabular}{lccccc}
\hline \hline$n$ & $E_{\text {diff }}(\mathrm{eV})$ & $E_{\mathrm{b}}(\mathrm{eV})$ & $d(\AA)$ & $m\left(\mu_{\mathrm{B}}\right)$ & $m_{\text {bare }}\left(\mu_{\mathrm{B}}\right)$ \\
\hline $1(\mathrm{a})$ & & -1.87 & & 3.31 & 3.24 \\
$2(\mathrm{~b})$ & 0.00 & 0.32 & 2.10 & $(2,38,-2.38)$ & $(2.79,2.79)$ \\
$2\left(\mathrm{~b}^{\prime}\right)$ & 0.73 & -0.67 & 2.03 & $(1.40,1.40)$ & $(2.72,2.72)$ \\
$3(\mathrm{c})$ & & 1.21 & $(2.22,2.22)$ & $(2.49,1.02,2.49)$ & $(3.04,2.96,3.04)$ \\
$4(\mathrm{~d})$ & 0.00 & 2.21 & $(2.18,2.18,2.18)$ & $(2.55,-0.29,0.29,-2.55)$ & $(3.08,2.88,2.88,3.08)$ \\
$4\left(\mathrm{~d}^{\prime}\right)$ & 0.20 & 2.32 & $(2.21,2.14,2.21)$ & $(2.35,-1.75,-1.75,2.35)$ & $(3.02,2.85,2.85,3.02)$ \\
\hline \hline
\end{tabular}




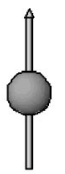

(a) $3.24\left(4.00 \mu_{\mathrm{B}}\right)$

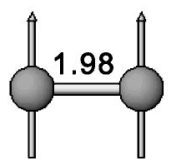

(b) 2.70

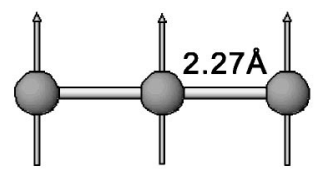

(c) $3.04 \quad 2.97 \quad\left(10.00 \mu_{\mathrm{B}}\right)$

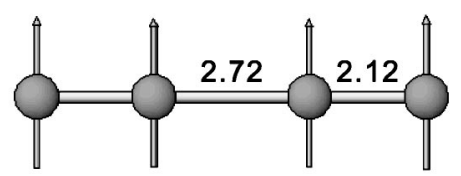

$\left(14.00 \mu_{\mathrm{B}}\right)$
FIG. 2. Magnetic moments and interatomic distances of free $\mathrm{Fe}_{n}$ linear chains in a vacuum; $n=$ (a) 1, (b) 2, (c) 3, and (d) 4. Magnetic moment of each atom $\left(\mu_{\mathrm{B}}\right)$ and the interatomic distance $(\AA)$ are indicated below and above the figure, respectively. Total magnetic moments of $\mathrm{Fe}$ chains are also indicated in parentheses.

pied antibonding orbital $h_{g}^{*}$ makes the capsule energetically unfavorable. In addition, a $g_{u}$-like state, which is partially occupied in the empty $\mathrm{C}_{20}$ cage, migrates below $E_{\mathrm{F}}$, and is fully occupied in $\mathrm{FeC}_{20}$. This state also makes the capsule unfavorable. On the spin-down side, the $h_{g}^{*}$ orbital lies above $E_{\mathrm{F}}$ and the $3 d$-electron population is almost 1 . The resulting magnetic moment of the Fe atom is almost $4 \mu_{\mathrm{B}}\left[(5-1) \mu_{\mathrm{B}}\right]$.

The induced magnetic moments on the $\mathrm{C}$ atoms also indicate another source of energetic unfavorableness because the induced magnetic moments originate from the existence of dangling bonds.

\section{B. $\mathrm{Fe}_{2} \mathrm{C}_{30}$}

The optimization shows that the structure of $\mathrm{Fe}_{2} \mathrm{C}_{30}$ keeps the initial structure as shown in Fig. 1(b). The interatomic distance of the Fe dimer is $2.10 \AA$, which is larger by $5 \%$ than that of the bare dimer $(1.98 \AA)$ in Fig. 2(b), and close to the interval of the zigzag circles $(2.16 \AA)$.

The radius of the SWNT varies from $2.07 \AA$ (at the end, next to the terminal pentagon) to $2.21 \AA$ (in the middle, next to the end for this case). The tendency for the radius to become maximum next to the ends is preserved for all the capsules as shown in Figs. 1(a)-1(d). This may be simply due to an effect of termination by the pentagon.

The magnetic moments of $2.38 \mu_{\mathrm{B}}$ in the Fe dimer align in antiparallel in contrast with the parallel magnetic moments both in the free dimer shown in Fig. 2(b) and in the fixed bare dimer indicated as $m_{\text {bare }}$ in Table I, row 2(b). The absolute values of the magnetic moments are similar to those of the bare dimer in antiparallel alignment $\left(2.50 \mu_{\mathrm{B}}\right)$.

Small magnetic moments from $0.10 \mu_{\mathrm{B}}$ (end) to $0.03 \mu_{\mathrm{B}}$ (middle) are induced on the surrounding $\mathrm{C}$ atoms in parallel with that of the $\mathrm{Fe}$ atom, so that the orientation changes at the middle of the cage and they align in antiparallel to each side of the capsule. The absolute values are less than half of those in $\mathrm{FeC}_{20}$. This implies that the $\mathrm{C}$ orbitals change from dangling-bond-like ones to $\pi$-bond-like ones, and that the hybridization between the $3 d$ orbitals of $\mathrm{Fe}$ and $2 s$ or $2 p$ orbitals of $\mathrm{C}$ is weakened, especially in the middle region.
The DOS and PDOS curves in Fig. 3(b) indicate a typical antiferromagnetic feature. Three of the main peaks of the PDOS (large peaks at $-8.1,-3.2$, and $-1.8 \mathrm{eV}$ ) are similar to those of $\mathrm{FeC}_{20}$ in Fig. 3(a). Moreover, these PDOSs include the component of only one site of Fe. That is, these parts are localized at both ends and hybridized independently with the $\mathrm{C}$ orbitals around the pentagon on each side. It is noticed that these peaks remain through all the capsules as shown in Figs. 3(a)-3(d). Meanwhile, the small PDOS between -8 and $-4 \mathrm{eV}$ consists of almost equal proportions of both sites of Fe. Then, these are coupling parts within the Fe dimer.

The highest occupied levels $e_{1}$ and $e_{2}$ in Fig. 3(b) are fully occupied. These peaks are mainly composed of $d_{x z}, d_{y z}$, and $d_{x y}, d_{x^{2}-y^{2}}$ orbitals, respectively. This configuration seems to be stable because the electronic structure around $E_{\mathrm{F}}$ is closed-shell-like, similarly to that of the ferrocene molecule $\mathrm{Fe}\left(\mathrm{C}_{5} \mathrm{H}_{5}\right)_{2}$, which realizes a stable closed-shell structure by satisfying the 18 -electron rule. ${ }^{40} e_{1}, e_{2}$, and their antibonding states $e_{1}^{*}$ and $e_{2}^{*}$ at $-2 \mathrm{eV}$ seem to correspond to the $e_{1 g}$ and $e_{2 g}$ states in ferrocene where $E_{\mathrm{F}}$ is located between the two states.

When the magnetic alignment is fixed in parallel, the interatomic distance of the $\mathrm{Fe}$ dimer shortens to $2.03 \AA$. As listed in Table I, row $2\left(\mathrm{~b}^{\prime}\right)$, the total energy is $0.73 \mathrm{eV}$ higher than that for the antiparallel alignment, whereas for the bare dimer the parallel alignment is lower in energy by $1.46 \mathrm{eV}$ than the antiparallel one. Furthermore, the binding energy $E_{\mathrm{b}}$ becomes negative $(-0.67 \mathrm{eV})$. Why are the magnetic moments of $\mathrm{Fe}_{2}$ in the cage of $\mathrm{C}_{30}$ stabilized in the antiparallel alignment? The answer is partially given by comparing electronic structures: As shown in the DOS for the parallel magnetic alignment in Fig. 3( $\left.\mathrm{b}^{\prime}\right)$, an antibonding orbital $e_{2}^{*}$ in the majority spin state migrates into the occupied states, and conversely a bonding orbital $e_{2}$ in the minority spin state rises up to the unoccupied states. Consequently, the total spin magnetic moment amounts to $4 \mu_{\mathrm{B}}$; however, the atomic moment on each $\mathrm{Fe}$ decreases to $1.4 \mu_{\mathrm{B}}$, resulting in a loss of atomic magnetic energy. Therefore, compared with the antiparallel alignment, the parallel alignment has the disadvantage of both the orbital energy around $E_{\mathrm{F}}$ and the intraatomic exchange energy of $\mathrm{Fe}$.

Another point of discussion is as follows. The parallel alignment in the bare dimer is stabilized by the ferromagnetic interaction that may be induced by the direct exchange interaction between $3 d$ electrons and the gain of kinetic energy from the electrons in minority spin states. For the dimer in the cage, there additionally exists an effective interaction between the magnetic moments through the cage, because of the orbital hybridization between each $\mathrm{Fe}$ atom and the $\mathrm{C}$ atoms of the cage. This hybridization induces a spin density on the $\mathrm{C}$ atoms that is parallel to the moment on the adjacent Fe. Within the electronic structure in the cage of $\mathrm{C}_{30}$, although the spin polarization is unfavorable apparently, the antiparallel spin density as illustrated in Fig. 1(b) may be more stable than the parallel one because of the energy gain from electron kinetics (chemical bonding energies). This effective interaction is a kind of superexchange interaction. ${ }^{41}$

\section{C. $\mathrm{Fe}_{\mathbf{3}} \mathbf{C}_{\mathbf{4 0}}$}

The three Fe atoms keep the linear shape with the interatomic distance of $2.22 \AA$ as shown in Fig. 1(c). This dis- 

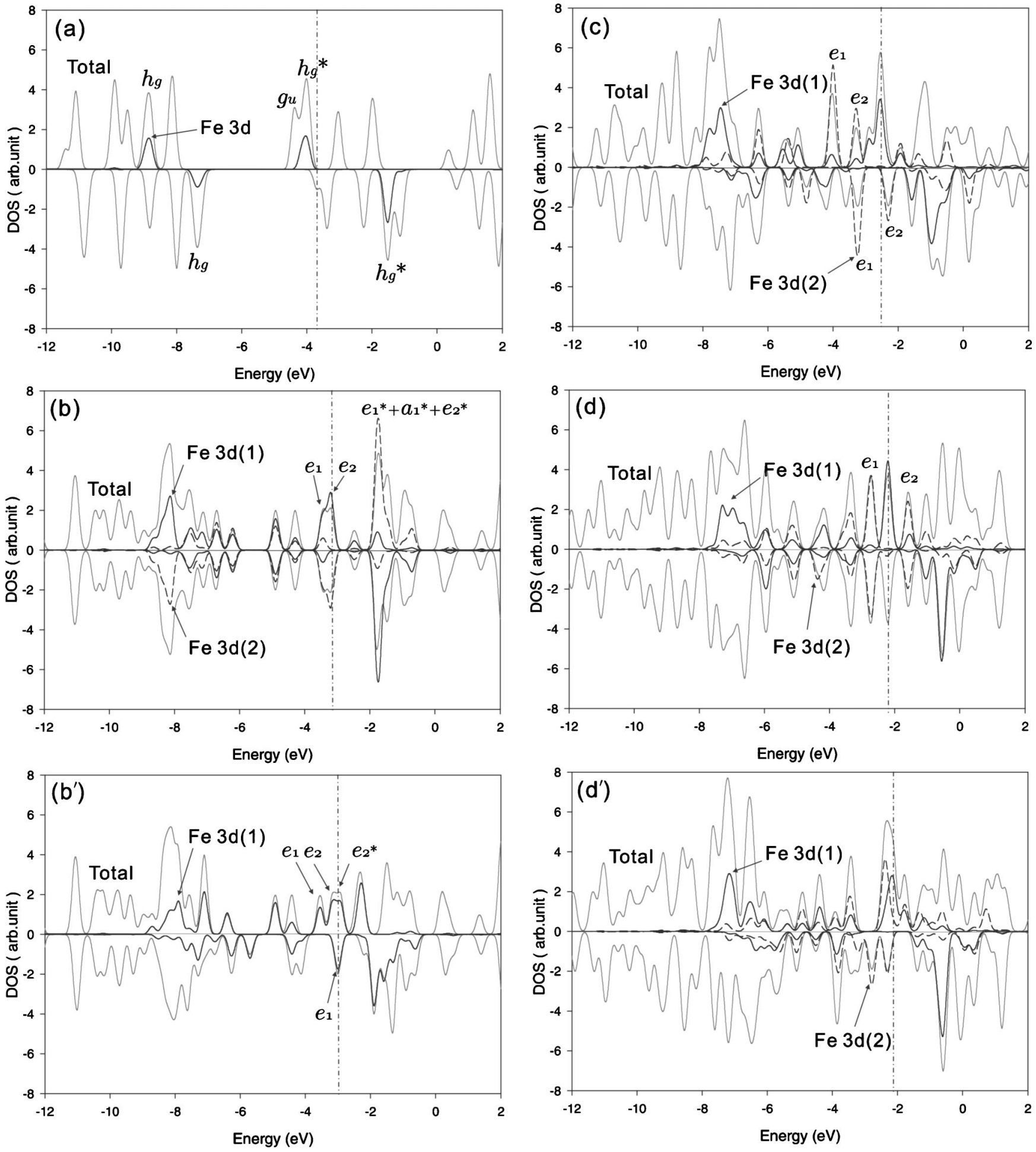

FIG. 3. Density of states of $\mathrm{Fe}_{n} \mathrm{C}_{10(n+1)}: n=$ (a) 1, (b) 2, (c) 3, and (d) 4. For each spin state (spin up, upper side; spin down, lower side), gray curves indicate the total density of states (DOS) and solid and dashed curves the partial density of states (PDOS) of Fe $3 d$ electrons for different atomic sites (multiplied by certain integers). The numbers in parentheses correspond to the atomic sites in Fig. 1. The vertical dash-dotted line indicates the highest occupied level. For $n=2$ and 4 , DOSs of other magnetic alignments are also shown as $\left(b^{\prime}\right)$ and $\left(\mathrm{d}^{\prime}\right)$.

tance is in the middle between that of the bare chain $(2.27 \AA)$ and the interval of the zigzag circle $(2.18 \AA)$. The most stable structure of bare $\mathrm{Fe}_{3}$ is not a linear chain but a triangle..$^{20,21,42}$ However in the SWNC, a slightly bent chain as an initial structure becomes not trigonal but linear without breaking the SWNC structure.

The magnetic moments of the $\mathrm{Fe}$ atoms align in parallel to each other. The magnetic moment at the ends is $2.49 \mu_{\mathrm{B}}$, 
similar to those of the $\mathrm{Fe}$ atoms in $\mathrm{Fe}_{2} \mathrm{C}_{30}$. The PDOS of the end $\mathrm{Fe}$ atom (1) (solid curve) in Fig. 3(c) indicates that the orbitals of the main peaks are localized at the ends, as in the smaller capsules in Figs. 3(a) and 3(b).

The magnetic moment at the center atom is $1.02 \mu_{\mathrm{B}}$. This value is very small in comparison with that of the free $\mathrm{Fe}_{3}$ chain in Fig. 2(c) or the bare chain in Table I, row 2(c). The PDOS of the center atom (2) (dashed curves) shows that both the large peaks $e_{1}$ and $e_{2}$ are below $E_{\mathrm{F}}$ in spin-up states while the peak $e_{2}$ is above $E_{\mathrm{F}}$ in the spin-down states. The difference yields $1 \mu_{\mathrm{B}}$ of spin magnetic moment in the center Fe.

These levels $e_{1}$ and $e_{2}$ have little or zero component of the end atoms (solid curve), so the $3 d$ electrons at the center atom are mainly combined with surrounding $\mathrm{C}$ atoms. The induced magnetic moment on the $\mathrm{C}$ atoms is very small in the middle of capsule, and the energy distribution of the PDOS (2) is narrower than that of PDOS (1); the main peaks of PDOS (2) exist between the well-split large peaks of PDOS (1). Then, the hybridization between $2 s$ and $2 p$ orbitals of $\mathrm{C}$ atoms and $3 d$ orbitals of the center Fe atom may be relatively weak. This may result in a weak antiferromagnetic interaction through the cage between neighboring Fe atoms, and the direct ferromagnetic interaction may be dominant.

Previous works employing the local-density approximation ${ }^{24,28}$ suggested that there is an antiferromagnetic interaction between the next nearest neighbor of $\mathrm{Fe}$ atoms in the bare linear $\mathrm{Fe}_{3}$ clusters, while this work using the generalized gradient approximation shows ferromagnetic configurations in both the bare and encapsulating $\mathrm{Fe}_{3}$ clusters.

\section{D. $\mathrm{Fe}_{4} \mathrm{C}_{50}$}

The four Fe atoms in Fig. 1(d) constitute a linear chain with almost equal intervals of $2.18 \AA$. Therefore, no dimerization occurs in $\mathrm{Fe}_{4} \mathrm{C}_{50}$. By contrast, the free four-atom chain separates into two dimers as shown in Fig. 2(d). Free chains with even number of atoms have a general tendency to dimerize, e.g., the Peierls distortion. ${ }^{27}$ But in the capsule, the structures of surrounding $\mathrm{C}$ atoms (the interval of the zigzag circles is $2.18 \AA$ ), as well as the antiparallel coupling described below, weaken the tendency to dimerization.

The magnetic moments of $\mathrm{Fe}$ atoms align in antiparallel each other. The absolute magnetic moments at the middle and end $\mathrm{Fe}$ atoms are $0.29 \mu_{\mathrm{B}} /$ atom and $2.55 \mu_{\mathrm{B}} /$ atom, respectively. The value of the latter is similar to that in $\mathrm{Fe}_{2} \mathrm{C}_{30}\left(2.38 \mu_{\mathrm{B}}\right)$ or $\mathrm{Fe}_{3} \mathrm{C}_{40}\left(2.49 \mu_{\mathrm{B}}\right)$.

The induced magnetic moments on the carbon cage align in the same direction for the Fe atoms at the ends, and in antiparallel along each zigzag circle in the middle region. However, the absolute value at the middle is very small, as in the middle of $\mathrm{Fe}_{3} \mathrm{C}_{40}$. Therefore, the induced magnetic moment on the carbon cage is due to a terminal effect where $s p^{3}$-like orbitals exist on pentagonal rings.

The PDOS of the end Fe atom (1) indicated by the solid curves in Fig. 3(d) is also similar to those of the smaller capsules. This means that these levels are still localized at the ends of the capsule. The PDOS of the middle $\mathrm{Fe}$ atoms (2) around $E_{\mathrm{F}}$ is similar to that in $\mathrm{Fe}_{3} \mathrm{C}_{40}$ if the $e_{2}$ level in the majority spin rises up to the unoccupied states. Because there is no occupation of the $e_{2}$ level, the magnetic moments of the inner $\mathrm{Fe}$ atoms are very small.

Another antiparallel magnetic alignment with only $0.2 \mathrm{eV}$ higher total energy exists as listed in Table I, row $4\left(\mathrm{~d}^{\prime}\right)$. In this alignment, a parallel magnetic moment of $1.75 \mu_{\mathrm{B}}$ in the central dimer is coupled antiparallel with the end atoms; the interatomic distances of the $\mathrm{Fe}$ atoms are $2.14 \AA$ in the dimer and $2.21 \AA$ between the central dimer and the end atoms. Then a weak dimerization appears and it brings parallel magnetic alignment with a relatively large magnetic moment.

The PDOS of the Fe atoms in Fig. 3(d') is still similar to those of the smaller capsules: the PDOS of the end atoms (1) has three localized peaks. The PDOS of the middle atoms (2) around $E_{\mathrm{F}}$ is similar to that in $\mathrm{Fe}_{3} \mathrm{C}_{40}$ if turned upside down, that is, there exist two large peaks in the majority spin state and one in the minority spin state, and the $3 d$ electrons of the middle atoms are combined, not with those of the end $\mathrm{Fe}$ atoms, but with $2 s$ and $2 p$ electrons of the surrounding $\mathrm{C}$ atoms.

We also have obtained a fully parallel magnetic alignment with $0.4 \mathrm{eV}$ higher energy. In this alignment, very small magnetic moments of $0.1 \mu_{\mathrm{B}}$ appear in the middle region.

\section{E. $\mathrm{FeC}_{48}$ and $\mathrm{Fe}_{2} \mathrm{C}_{48}$}

To compare the structures, positions, and magnetic moments of Fe atoms in thicker cages with those in the $(5,0)$ SWNC discussed above, we calculate the electronic states and optimize the structures of the capsules $\mathrm{FeC}_{48}$ and $\mathrm{Fe}_{2} \mathrm{C}_{48}$.

Figure 4(a) shows two side views of structures of $\mathrm{FeC}_{48}$ with three different $\mathrm{Fe}$ sites. The magnetic moments of $\mathrm{Fe}$ atom (1) and those induced on C atoms are also indicated on the lower side. Details such as the total energy differences, binding energies, positions, and magnetic moments of the Fe atom are listed in Table II.

We obtain three stable positions of the Fe atom. When the atom is located on a plane across the zigzag ring as the initial position, the atom settles on the center axis ( $z$ axis) of the capsule after optimization, even if the initial position is deviates by $1.6 \AA$ from the axis. This optimized position is shown as site (1) in the figure. In contrast, when the atom is located on the plane including the centers of hexagons $(x-y$ plane), the $\mathrm{Fe}$ atom settles at a position deviating by about $1.5 \AA$ from the center or $1.6-1.8 \AA$ from the capsule wall. These are shown as the edge site (1') and hollow site (1") in the figure. The total-energy calculation indicates that the center site (1) is more favorable by 0.37 and $0.48 \mathrm{eV}$ than the deviated sites $\left(1^{\prime}\right)$ and $\left(1^{\prime \prime}\right)$, respectively. The binding energy defined by $\left(E_{\mathrm{Fe}}+E_{\mathrm{C}_{48}}\right)-E_{\mathrm{FeC}_{48}}$ is also largest for the $\mathrm{Fe}$ site (1).

In comparison of the site $\left(1^{\prime}\right)$ with $\left(1^{\prime \prime}\right)$, the energetic favorableness agrees with the previous results for the infinite $(8,0)$ SWNT.${ }^{14}$ The distance between the Fe atom and carbon wall also agrees with the previous results $1.7-1.9 \AA$. However, the binding energy for the site (1) is much larger than those of the sites $\left(1^{\prime}\right)$ and $\left(1^{\prime \prime}\right)$, and the distance from the carbon wall is much larger than their results. This disagreement may be caused by the terminal octagon next to the 


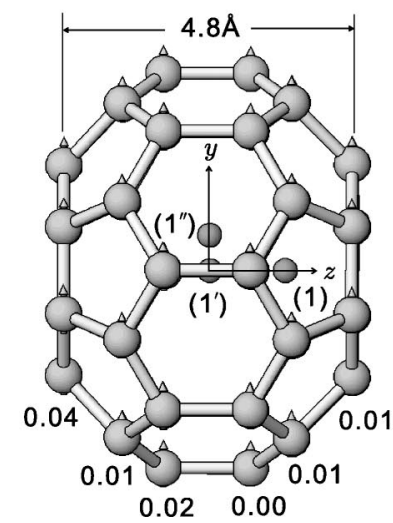

(a)

(1) $2.64\left(4.00 \mu_{\mathrm{B}}\right)$

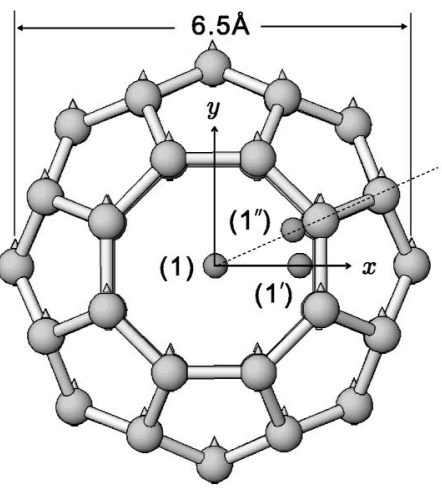

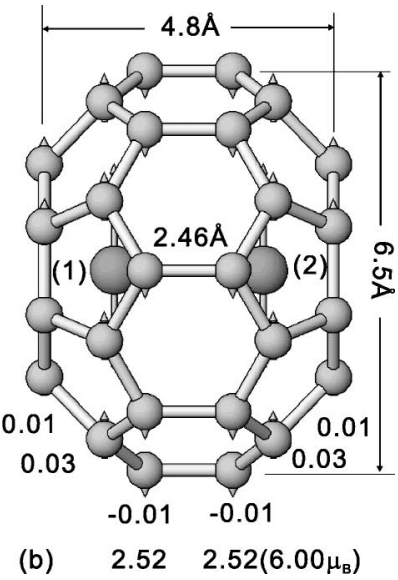

(b) $\quad 2.52 \quad 2.52\left(6.00 \mu_{\mathrm{B}}\right)$

FIG. 4. Structures and magnetic moments of $\mathrm{Fe}_{n} \mathrm{C}_{48}$; (a) two side views for $n=1$ and (b) for $n=2$. The magnetic moments $\left(\mu_{\mathrm{B}}\right)$ of Fe atoms are listed below the figures. The net magnetic moment of the whole capsule is shown in parentheses. The magnetic moments of the $\mathrm{C}$ cages are indicated on the lower side. The sites $\left(1^{\prime}\right)$ and $\left(1^{\prime \prime}\right)$ are slightly higher-energy sites of the Fe atom. Details are listed in Table II.

zigzag ring. Then it is suggested that Fe atoms may be located not along the center axis but near the $\mathrm{C}-\mathrm{C}$ bonds in an infinite nanotube, where no terminal effect exists.

The magnetic moment of an $\mathrm{Fe}$ atom at each site is not as large as that of a bare atom, and becomes smaller in order of the binding energy, that is, the degree of interaction between $\mathrm{Fe}$ and $\mathrm{C}$ atoms.

Figure 4(b) shows the structures and magnetic moments of the $\mathrm{Fe}_{2} \mathrm{C}_{48}$ capsule. The positions and magnetic moments of the Fe dimer are listed in Table II, row 2(b). The Fe dimer aligns along the center axis of the capsule, like the dimer in the thinner capsule $\mathrm{Fe}_{2} \mathrm{C}_{30}$ and like the $\mathrm{Fe}$ atom in $\mathrm{FeC}_{48}$, even if the dimer is put $1.6 \AA$ off from the center axis as the initial atomic position. Fagan et al. reported that the infinite Fe chain in an $(8,0)$ SWNT has a zigzag structure deviating from the center axis. ${ }^{15}$ This structural difference may be caused by a terminal effect similar to that observed for $\mathrm{FeC}_{48}$ in Fig. 4, sites (1) and $\left(1^{\prime}\right)$.

The parallel magnetic arrangement is the lowest, unlike in $\mathrm{Fe}_{2} \mathrm{C}_{30}$. However, we have obtained another slightly higherenergy state with antiparallel magnetic arrangement as shown in Table II, row 2( $\left.b^{\prime}\right)$. The total energy difference is only $0.13 \mathrm{eV}$, which is smaller than that for $\mathrm{Fe}_{2} \mathrm{C}_{30}(0.73 \mathrm{eV}$

TABLE II. Total-energy differences $E_{\text {diff }}$, binding energies $E_{\mathrm{b}}$, coordinates of $\mathrm{Fe}$ atoms $(x, y, z)$, and magnetic moments of $\mathrm{Fe}$ atoms, $m$, for $\mathrm{Fe}_{n} \mathrm{C}_{48}$ capsules. The numbers 1-1" correspond to the $\mathrm{Fe}$ sites for $\mathrm{FeC}_{48}$ in Fig. 4(a). Magnetic moments of bare Fe and $\mathrm{Fe}_{2}$ with the same interatomic distances as those in the cages, $m_{\text {bare }}$, are also listed as references. Row $2\left(b^{\prime}\right)$ shows the higher-energy state in the antiferromagnetic arrangement.

\begin{tabular}{lccccc}
\hline \hline$n$ & $E_{\text {diff }}(\mathrm{eV})$ & $E_{\mathrm{b}}(\mathrm{eV})$ & $(x, y, z)(\AA)$ & $m\left(\mu_{\mathrm{B}}\right)$ & $m_{\text {bare }}\left(\mu_{\mathrm{B}}\right)$ \\
\hline $1(\mathrm{a})$ & 0.00 & 1.90 & $(0.00,0.00,1.28)$ & 2.64 & 3.24 \\
$1^{\prime}(\mathrm{a})$ & 0.37 & 1.67 & $(1.47,0.00,0.00)$ & 2.75 & 3.24 \\
$1^{\prime \prime}(\mathrm{a})$ & 0.49 & 1.45 & $(1.36,0.59,0.00)$ & 2.84 & 3.24 \\
$2(\mathrm{~b})$ & 0.00 & 2.62 & $(0.00,0.00, \pm 1.23)$ & 2.52 & 3.15 \\
$2\left(\mathrm{~b}^{\prime}\right)$ & 0.13 & 2.67 & $(0.00,0.00, \pm 1.25)$ & \pm 2.47 & 3.16 \\
\hline \hline
\end{tabular}

in Table I). Furthermore, the binding energy $E_{\mathrm{b}}$ is larger in antiparallel coupling rather than in the parallel one. This fact suggests that there exists some energetic favorableness of the antiferromagnetic arrangement due to the superexchange interaction assisted with the terminal effect.

The positions of $\mathrm{Fe}$ atoms for parallel and antiparallel magnetic arrangements are similar to each other and close to that in $\mathrm{FeC}_{48}$. So the interatomic distances $(\sim 2.5 \AA)$ are larger than those in $\mathrm{Fe}_{2} \mathrm{C}_{30}(\sim 2.1 \AA)$ or in the free $\mathrm{Fe}_{2}$ dimer $(\sim 2.0 \AA)$, and the interaction between $\mathrm{Fe}$ atoms is not very large.

Figure 5 shows the DOS and PDOS of Fe $3 d$ electrons, (a) for $\mathrm{FeC}_{48}$, and (b) and ( $\left.\mathrm{b}^{\prime}\right)$ for $\mathrm{Fe}_{2} \mathrm{C}_{48}$ with the ferromagnetic and antiferromagnetic arrangements, respectively. In comparison with Figs. 3(a) and $3\left(b^{\prime}\right)$, it is obviously found that no large separation of the $3 d$ PDOS appears in $\mathrm{Fe}_{n} \mathrm{C}_{48}$ : there exists one large peak of spin-up states at -4 to $-5 \mathrm{eV}$ for $\mathrm{Fe}_{n} \mathrm{C}_{48}$ while two separated peaks appear at -8 to -9 and -3 to $-4 \mathrm{eV}$ for $\mathrm{Fe}_{n} \mathrm{C}_{10(n+1)}$. This fact indicates that no strong bonding and antibonding states between Fe and terminal $\mathrm{C}$ atoms exist. As a result, there appears little energetic unfavorableness due to the existence of the occupied antibonding states, in contrast with the results in Figs. 3(a) and 3(b').

From the results above, the interaction between the $\mathrm{Fe}$ atom and the terminal ring in $\mathrm{C}_{48}$ seems to be not so large as in the thinner $\mathrm{C}_{10(n+1)}$. However, it may play a certain role in the settlement of $\mathrm{Fe}$ atoms along the center axis and the induction of the antiferromagnetic interaction.

\section{DISCUSSION AND CONCLUSIONS}

Linear Fe chains were introduced into carbon cages. It was found from the results for $\mathrm{Fe}_{n} \mathrm{C}_{10(n+1)}(n=3,4)$ that the end and middle $\mathrm{Fe}$ atoms had different hybridizations with the cage; regardless of the weak hybridization for middle Fe atoms, its magnetization was suppressed and the strong hybridization for the end atoms did not break the atomic magnetization. The latter feature gives rise to the energy level of the majority spin state, whose wave function is extended to 

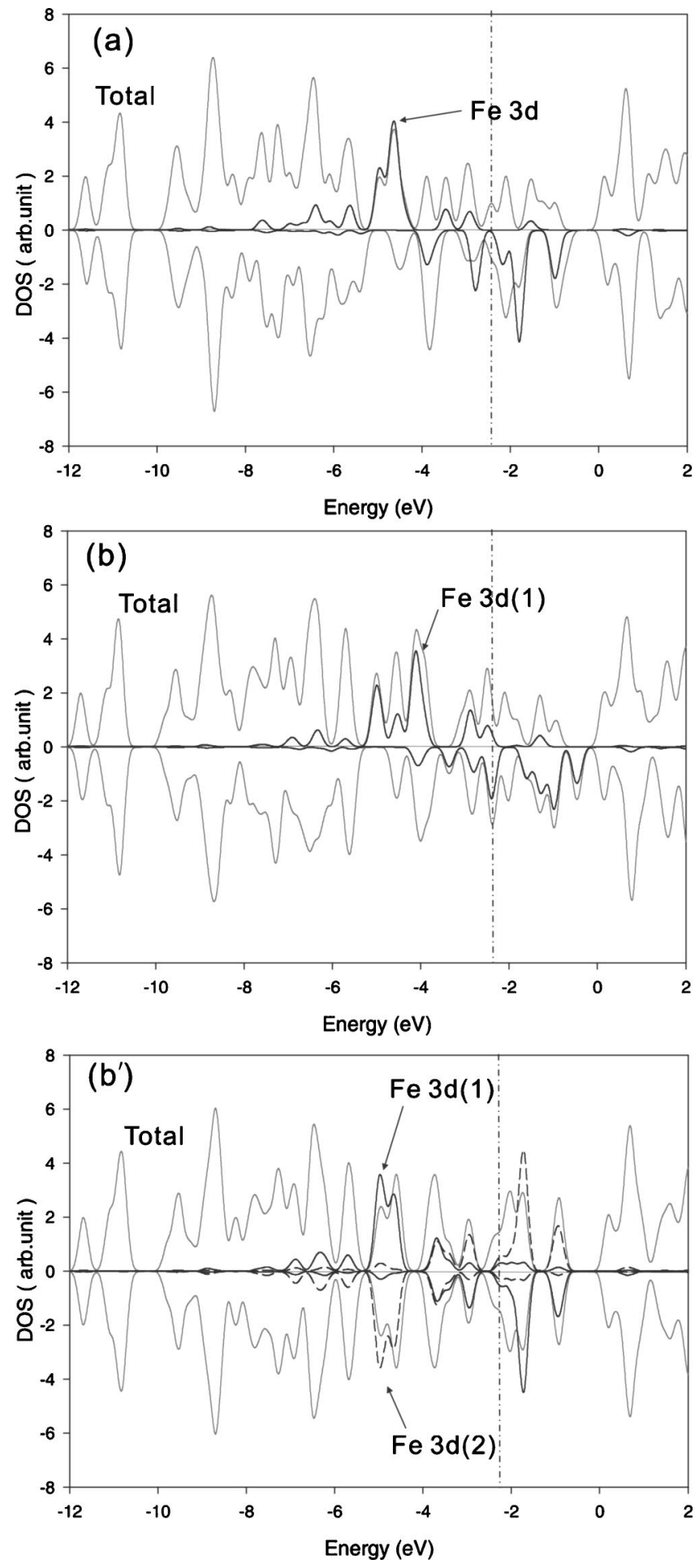

FIG. 5. Density of states of $\mathrm{Fe}_{n} \mathrm{C}_{48} ; n=$ (a) 1 and (b) 2. Gray curves indicate the total density of states (DOS), solid and dashed curves the partial density of states (PDOS) of Fe $3 d$ electrons. The numbers in parentheses correspond to the atomic sites in Fig. 4 and Table II. The vertical dash-dotted line indicates the highest occupied level. For $n=2$, the DOS of antiparallel magnetic alignments is also shown as $\left(b^{\prime}\right)$. the cage due to the hybridization with $s p^{3}$-like orbitals. However, the feature itself does not imply that stability of the local atomic configuration is guaranteed, and our totalenergy calculations show that the capsules stabilize by lengthening $(n>1)$ and by thickening $\left(\mathrm{C}_{48}\right)$.

The magnetic configuration of the middle $\mathrm{Fe}$ atoms is sensitive to the atomic configuration. This feature appears in a variety of low-lying energy configurations for $\mathrm{Fe}_{4} \mathrm{C}_{50}$. The sensitivity of electronic states related to the middle $\mathrm{Fe}$ atoms is attributed to two comparable interactions with the nearest neighbors of the Fe atom and carbon atoms in the zigzag ring. The hybridization with carbon could be enhanced by a resonance effect; the energy levels of the $\pi$ orbital in the carbon cage exist in the same energy range as the $3 d$ orbitals from $\mathrm{Fe}$ atoms.

Our results indicate that the carbon cages do not destroy the magnetism of linear Fe clusters, although the magnetism of $\mathrm{Fe}$ atoms has never been enhanced due to the hybridization, and the antiferromagnetic configurations have been favored due to the superexchange interaction through the cage.

For $\mathrm{Fe}_{4} \mathrm{C}_{50}$ and $\mathrm{Fe}_{2} \mathrm{C}_{30}$, some parallel magnetic alignments appear when dimerization or shortening of the interatomic distance occurs as described in Secs. III B and III D. It is expected that aggregation or cohesion of $\mathrm{Fe}$ atoms, such as shortening the interatomic distance or increasing the coordination number, brings a parallel magnetic alignment with a certain magnetic moment. Yang et al. have obtained a parallel magnetic moment of $2.34 \mu_{\mathrm{B}}$ per Fe atom in an infinite system $\mathrm{Fe}_{6} \mathrm{C}_{36}$ [a triangle antiprism of $\mathrm{Fe}$ in $\left.(9,0) \mathrm{SWNT}\right] .^{13}$

In conclusion, the structures and magnetism of $\mathrm{Fe}_{n}$ chains in tubal carbon nanocapsules $\mathrm{C}_{10(n+1)}$ and $\mathrm{C}_{48}$ are calculated by means of noncollinear first-principles molecular dynamics. The calculations show that $\mathrm{Fe}_{n} \mathrm{C}_{10(n+1)}$, which is energetically unfavorable for the globular molecule, $n=1$, becomes favorable on increasing the cage length, and that the $\mathrm{Fe}_{n}$ chains keep a linear structure along the center axis of the $\mathrm{C}_{10(n+1)}$ and $\mathrm{C}_{48}$ cages. The magnetic moments align in antiparallel for $n=2$ and 4 in the $\mathrm{C}_{10(n+1)}$ cage, while those of bare chains align in parallel. Antiparallel magnetic alignment also appears in $\mathrm{Fe}_{2} \mathrm{C}_{48}$ with a small total energy difference. Small magnetic moments are induced on $\mathrm{C}$ atoms with the same orientations as those on adjacent $\mathrm{Fe}$ atoms. For both the cages $\mathrm{C}_{10(n+1)}$ and $\mathrm{C}_{48}$, the terminal carbon rings play a certain role in the settlement of $\mathrm{Fe}$ atoms along the center axis and the favorableness of antiferromagnetic arrangements.

The results of the present work imply that carbon cages could be effective supporting materials for ferromagnetic clusters. The structural and magnetic properties described here will give knowledge about the boundaries or terminals of nanotubes even in thicker and longer finite systems.

\section{ACKNOWLEDGMENT}

This work is supported by a Grant-in-Aid for Scientific Research of JSPS, No. 13640328. 
${ }^{1}$ M. S. Dresselhaus, G. Dresselhaus, and P. Avouris, Carbon Nanotubes (Springer, Berlin, 2001).

${ }^{2}$ K. Tsukagoshi, B. W. Alphenaar, and H. Ago, Nature (London) 401, 572 (1999).

${ }^{3}$ X. X. Zhang, G. H. Wen, S. Huang, L. Dai, R. Gao, and Z. L. Wang, J. Magn. Magn. Mater. 231, L9 (2001).

${ }^{4}$ A. M. Cassell, W. A. Scrivens, and J. M. Tour, Proc.Electrochem. Soc. 95-10, 549 (1995).

${ }^{5}$ X. Zhao, S. Inoue, M. Jinno, T. Suzuki, and Y. Ando, Chem. Phys. Lett. 373, 266 (2003).

${ }^{6}$ M. Sawamura, Y. Kuninobu, M. Toganoh, Y. Matsuo, M. Yamanaka, and E. Nakamura, J. Am. Chem. Soc. 124, 9354 (2002).

${ }^{7}$ Y. Zhang, N. W. Franklin, R. J. Chen, and H. Dai, Chem. Phys. Lett. 331, 35 (2000).

${ }^{8}$ M. Monthioux, Carbon 40, 1809 (2002).

${ }^{9}$ B. C. Satishkumar, A. Govindaraj, P. Vanitha, A. K. Raychaudhuri, and C. N. R. Rao, Chem. Phys. Lett. 362, 301 (2002).

${ }^{10}$ T. Mühl, D. Elefant, A. Graff, R. Kozhuharova, A. Leonhardt, I. Mönch, M. Ritschel, P. Simon, S. Groudeva-Zotova, and C. M. Schneiderarb, J. Appl. Phys. 93, 7894 (2003).

${ }^{11}$ R. Kozhuharova, M. Ritschel, D. Elefant, A. Graff, A. Leonhardt, I. Möncha, S. G.-Z. T. Mühl, and C. M. Schneider, Appl. Surf. Sci. 238, 355 (2004).

${ }^{12}$ C. Yang, J. Zhao, and J. Lu, Phys. Rev. B 66, 041403 (2002).

${ }^{13}$ C. Yang, J. Zhao, and J. Lu, Phys. Rev. Lett. 90, 257203 (2003).

${ }^{14}$ S. B. Fagan, R. Mota, A. J. R. da Silva, and A. Fazzio, Phys. Rev. B 67, 205414 (2003).

${ }^{15}$ S. B. Fagan, R. Mota, A. J. R. da Silva, and A. Fazzio, Microelectron. J. 34, 481 (2003).

${ }^{16}$ C. Binns, S. H. Baker, A. M. Keen, S. N. Mozley, C. Norris, H. S. Derbyshire, and S. C. Bayliss, Phys. Rev. B 53, 7451 (1996).

${ }^{17}$ D. M. Duffy and J. A. Blackman, Phys. Rev. B 58, 7443 (1998).

${ }^{18}$ P. Kruger, M. Taguchi, J. C. Parlebas, and A. Kotani, Phys. Rev. B 59, 15093 (1999).

${ }^{19}$ J. W. Mintmire, B. I. Dunlap, and C. T. White, Phys. Rev. Lett. 68, 631 (1992).
${ }^{20}$ M. Castro and D. R. Salahub, Phys. Rev. B 49, 11842 (1994).

${ }^{21}$ O. Diéguez, M. M. G. Alemany, C. Rey, P. Ordejón, and L. J. Gallego, Phys. Rev. B 63, 205407 (2001).

${ }^{22}$ I. L. M. Billas, A. Châtelain, and W. A. de Heer, Science 265, 1682 (1994)

${ }^{23}$ S. E. Apsel, J. W. Emmert, J. Deng, and L. A. Blooomfeld, Phys. Rev. Lett. 76, 1441 (1996).

${ }^{24}$ T. Oda, A. Pasquarello, and R. Car, Phys. Rev. Lett. 80, 3622 (1998).

${ }^{25}$ N. Fujima, Eur. Phys. J. D 16, 185 (2001).

${ }^{26}$ N. Fujima, J. Phys. Soc. Jpn. 71, 1529 (2002).

${ }^{27}$ N. Fujima and T. Oda, Eur. Phys. J. D 24, 89 (2003).

${ }^{28}$ D. Hobbs, G. Kresse, and J. Hafner, Phys. Rev. B 62, 11556 (2000).

${ }^{29}$ O. Ivanov and V. P. Antropov, J. Appl. Phys. 85, 4821 (1999).

${ }^{30}$ X. Zhao, Y. Liu, S. Inoue, T. Suzuki, R. O. Jones, and Y. Ando, Phys. Rev. Lett. 92, 125502 (2004).

${ }^{31}$ R. Car and M. Parrinello, Phys. Rev. Lett. 55, 2471 (1985).

${ }^{32}$ J. Kübler, K.-H. Hock, J. Sticht, and A. R. Williams, J. Phys. F: Met. Phys. 18, 469 (1988).

${ }^{33}$ D. Vanderbilt, Phys. Rev. B 41, 7892 (1990).

${ }^{34}$ A. Pasquarello, K. Laasonen, R. Car, C. Lee, and D. Vanderbilt, Phys. Rev. Lett. 69, 1982 (1992)

${ }^{35}$ J. P. Perdew, J. A. Chevary, S. H. Vosko, K. A. Jackson, M. R. Pederson, D. J. Singh, and C. Fiolhais, Phys. Rev. B 46, 6671 (1992).

${ }^{36}$ K. Knöpfle, L. M. Sandratskii, and J. Kübler, Phys. Rev. B 62 , 5564 (2000).

${ }^{37}$ K. Laasonen, A. Pasquarello, R. Car, C. Lee, and D. Vanderbilt, Phys. Rev. B 47, 10142 (1993).

${ }^{38}$ T. Oda, J. Phys. Soc. Jpn. 71, 519 (2002).

${ }^{39}$ T. Oda and A. Pasquarello, Phys. Rev. B 70, 134402 (2004).

${ }^{40}$ F. A. Cotton, G. Wilkinson, C. A. Murillo, and M. Bochmann, Advanced Inorganic Chemistry, 6th ed. (Wiley, Berlin, 1999).

${ }^{41}$ P. W. Anderson, Phys. Rev. 115, 2 (1959).

${ }^{42}$ G. L. Gutsev, S. N. Khanna, and P. Jena, Phys. Rev. B 62, 1604 (2000). 